\title{
Diâmetro do trado e número de amostras para quantificação do banco de sementes de arroz-vermelho do solo
}

\author{
Core diameter and number of samples to estimative the red rice seed bank \\ Sérgio Luiz de Oliveira Machado ${ }^{\mathrm{I} *}$ Luis Antonio de Avila" Enio Marchesan ${ }^{\mathrm{II}}$ Lindolfo Storck ${ }^{\mathrm{II}}$ \\ Geovane Boschmann Reimche ${ }^{\mathrm{I}}$ Paulo Fabrício Sachet Massoni ${ }^{\mathrm{II}}$ Heins KummerII \\ Luiz Felipe Thomás ${ }^{\mathrm{IV}}$
}

\begin{abstract}
Com o objetivo de determinar o diâmetro ideal do RESUMO trado e o número de amostras necessárias para quantificar o banco de sementes de arroz-vermelho, foram realizados levantamentos em lavouras de arroz irrigado, no Município de Santa Maria, Rio Grande do Sul (RS), Brasil. O experimento foi conduzido em três áreas de $400 \mathrm{~m}^{2}(20 \times 20 \mathrm{~m})$, com diferentes níveis de infestação, sendo considerado nível baixo, médio e alto, respectivamente, 71, 282 e 498 sementes de arrozvermelho por $\mathrm{m}^{2}$, coletadas com trados de 5, 10 e $15 \mathrm{~cm}$ de diâmetro. Os resultados mostraram que, quanto maior o diâmetro do trado, menor foi o $\mathrm{CV}$ entre as amostras. $\mathrm{O}$ trado com $10 \mathrm{~cm}$ de diâmetro foi mais funcional e necessitou de 280, 55 e $31(D=20 \%) ; 33$, nove e seis $(D=60 \%)$; e 13, quatro e três $(D=100 \%)$ amostras de solo, respectivamente, para as infestações de 71, 282 e 498 sementes de arroz-vermelho por $\mathrm{m}^{2}$. O número de amostras de solo necessário para melhor estimar populações de sementes de arroz-vermelho é variável com o número médio de sementes no solo e com a precisão desejada.
\end{abstract}

Palavras-chave: fitossociologia, nível de infestação, Oryza sativa, tamanho da amostra, variabilidade.

\section{ABSTRACT}

Aiming to estimate the number of samples, and the diameter of soil sampler ideal for red rice infestation level, a irrigated rice field survey has been conducted in Santa Maria, Rio Grande do Sul (RS), Brazil, The experiment was conducted in three areas with $400 \mathrm{~m}^{2}(20 \times 20 \mathrm{~m})$ with different red rice infestation levels considering low, medium and high infestation levels with 71, 282 and 498 red rice seeds per $m^{2}$, respectively, sample with 5, 10 and $15 \mathrm{~cm}$ core diameter. The results showed that with larger core diameter the samples coefficient variation among samples was smaller. The core of $10 \mathrm{~cm}$ is more functional, been necessary 280, 55 and $31(D=20 \%), 33,9$ and $6(D=60 \%)$ and 13, 4 and $3(D=100 \%)$ soil samples for the infestation levels 71, 282 and 498 red rice seeds per $m^{2}$ respectively. The number of soil samples necessary to estimate the red rice seed bank is variable with the infestation level and the desirable precision.

Key works: confidence interval, infestation level, Oryza sativa, phytosociological, variability.

O termo "banco de sementes" é usado para descrever o total de sementes e outras estruturas de propagação (rizomas, estolões e tubérculos) das plantas presentes no solo (CARMONA, 1992), bem como para conduzir estudos fotossociológicos sobre a dinâmica populacional, aspectos muito importantes para estabelecer um programa de manejo das plantas daninhas (AMBRÓSIO et al., 2004).

O banco de sementes é uma importante ferramenta na predição de futuras populações de plantas daninhas na área. Para tal, é necessário, para uma boa precisão, que o número de amostras e a

IDepartamento de Defesa Fitossanitária, Centro de Ciências Rurais (CCR), Universidade Federal de Santa Maria (UFSM), 97105900, Santa Maria, RS, Brasil. E-mail: smachado@smail.ufsm.br. *Autor para correspondência.

IDepartamento de Fitotecnia, UFSM, Santa Maria, RS, Brasil.

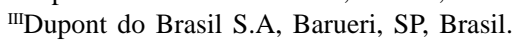

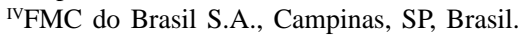


quantificação de sementes por amostra sejam representativos (ROBERTS \& RICKETTS, 1979). No solo, as sementes de plantas daninhas seguem o modelo da distribuição de Poisson; dessa forma, para um grau aceitável de precisão na estimativa do número mínimo de sementes coletadas, o tamanho da amostra pode ser grande (AMBRÓSIO et al., 1997). Assim, este estudo teve por objetivo determinar, para uma boa precisão, o diâmetro ideal do trado e o número de amostras no solo necessárias para quantificar o número de sementes de arroz-vermelho presentes no banco de sementes do solo, de acordo com os níveis iniciais de infestação.

Foram coletadas amostras de solo em lavouras de arroz irrigado do Município de Santa Maria, Rio Grande do Sul (RS), Brasil, em área de 20x20m, com três níveis de infestação de sementes de arroz-vermelho, sendo considerado o nível baixo $=71$ sementes de arroz vermelho por $\mathrm{m}^{2}$, o nível médio = 282 sementes de arroz vermelho por $\mathrm{m}^{2}$ e o nível alto $=498$ sementes por $\mathrm{m}^{2}$. Em cada área, foram coletadas 20 amostras de solo com cada um dos três trados (5, 10 e 15cm de diâmetro) na profundidade de $0-15 \mathrm{~cm}$. Em seguida, em laboratório, as sementes de arroz-vermelho foram separadas do solo e contadas, e os dados foram convertidos para número de sementes por $\mathrm{m}^{2}$.

Para cada nível de infestação de arrozvermelho, foram estimados a média, o coeficiente de variação e o tamanho da amostra necessária para uma semiamplitude do intervalo de confiança (D) a 95\% de probabilidade de erro igual a 20, 60 e 100\% da média amostral. Para cada diâmetro de trado, nos três diferentes níveis de infestação de arroz-vermelho, as médias do número de sementes por $\mathrm{m}^{2}$ foram comparadas pelo teste "t", em nível de 5\% de probabilidade de erro. Também foi estimado o coeficiente de correlação linear entre o diâmetro do trado com as médias do número de sementes de arroz- vermelho encontradas para cada nível de infestação e o coeficiente de variação. Para os testes de hipótese e estimativa do tamanho da amostra, os dados foram transformados para valores da $\sqrt{x}$. O tamanho da amostra (n) foi estimado por meio da fórmula: $\mathrm{n}=\mathrm{t}^{2} \mathrm{CV}^{2} /$ $\mathrm{D}^{2}$, em que $\mathrm{t}=$ valor tabelado da distribuição de $\mathrm{t}$ com $5 \%$ de probabilidade de erro (bilateral) e inicialmente com $n-1=19$ graus de liberdade; $\mathrm{D}=$ semiamplitude do intervalo de confiança igual a 20, 60 e 100\%; e $\mathrm{CV}=$ coeficiente de variação da amostra (STEEL et al, 1997).

Entre os níveis de infestação, houve diferença no número de sementes de arroz-vermelho para os níveis de infestações médio e baixo (Tabela 1). Para a infestação alta, houve correlação linear significativa entre o diâmetro do trado e as médias do nível de infestação. Além disso, o trado com menor diâmetro superestimou a quantidade de sementes no solo, corroborando VIDOTTO et al. (2001), que obtiveram resultados semelhantes na predição do banco de sementes com nível aceitável de precisão. Os maiores CV estão associados com as menores médias do número de sementes.

O CV também foi inversamente relacionado com o diâmetro do trado para os níveis de infestações baixo e médio (Tabela 2). Todavia, com alta infestação de arroz-vermelho, não foi verificada essa correlação, indicando que nesses locais as amostras de solo para a quantificação do banco de sementes foram representativas com qualquer um dos diâmetros de trados estudados. Dentre os critérios usados na escolha do diâmetro do trado, deve-se considerar, além do número de amostras para um D aceitável,a praticidade da coleta e também o tempo necessário para separar as sementes do solo. Assim, para $\mathrm{D}=60 \%$ e um local com baixo nível de infestação de arroz-vermelho, devem ser coletadas 67, 33 e nove amostras de solo, respectivamente, com trados de 5,10 e $15 \mathrm{~cm}$ de

Tabela 1 - Número de sementes de arroz-vermelho por $\mathrm{m}^{2}$ em função do diâmetro do trado e do nível de infestação e coeficiente de correlação linear (r) entre o diâmetro do trado e número de sementes. Santa Maria, RS. 2009.

\begin{tabular}{llll}
\hline & & & \\
Diâmetro do trado (cm) & Baixo & Médio & Alto \\
\hline 5 & $76 \mathrm{c}^{*}$ & $331 \mathrm{~b}$ & $662^{\mathrm{ns}}$ \\
10 & $83 \mathrm{a}$ & $153 \mathrm{~b}$ & 465 \\
15 & $54 \mathrm{~b}$ & $362 \mathrm{a}$ & 368 \\
Média & 71 & 282 & 498 \\
$\mathrm{r}$ & $-0,74^{\mathrm{ns}}$ & $0,13^{\mathrm{ns}}$ & $-0,98^{1}$ \\
\hline
\end{tabular}

*Na coluna, médias não seguidas pela mesma letra minúscula diferem pelo teste t, em nível de 5\% de probabilidade de erro.

${ }^{1}$ Significativo em nível de $5 \%$ de probabilidade de erro. 
Tabela 2 - Tamanho de amostra para semiamplitude do intervalo de confiança (D, em percentagem da média) do número de sementes por m² em função do diâmetro do trado e do nível de infestação de arroz-vermelho; coeficiente de variação do número de sementes de arroz-vermelho por $\mathrm{m}^{2}$ e coeficiente de correlação linear (r) entre diâmetro do trado e coeficientes de variação. Santa Maria, RS. 2009.

\begin{tabular}{|c|c|c|c|c|c|c|c|}
\hline \multirow{3}{*}{ Diâmetro do trado $(\mathrm{cm})$} & \multirow{3}{*}{$\mathrm{D}(\%)$} & \multicolumn{3}{|c|}{ Tamanho da amostra } & \multicolumn{3}{|c|}{ CV (\%) } \\
\hline & & --------- & -------- & --Níve & stação----. & ----------. & --------- \\
\hline & & Baixo & Médio & Alto & Baixo & Médio & Alto \\
\hline & 20 & 599 & 122 & 62 & & & \\
\hline \multirow[t]{3}{*}{5} & 60 & 67 & 16 & 9 & 79 & 112 & 247 \\
\hline & 100 & 26 & 7 & 5 & & & \\
\hline & 20 & 280 & 55 & 31 & & & \\
\hline \multirow[t]{3}{*}{10} & 60 & 33 & 9 & 6 & 55 & 74 & 169 \\
\hline & 100 & 13 & 4 & 3 & & & \\
\hline & 20 & 65 & 18 & 51 & & & \\
\hline \multirow[t]{2}{*}{15} & 60 & 9 & 4 & 8 & 71 & 39 & 81 \\
\hline & 100 & 5 & 3 & 4 & & & \\
\hline $\mathrm{r}$ & & & & & $-0,99 *$ & $-0,99 *$ & $-0,33^{\mathrm{ns}}$ \\
\hline
\end{tabular}

*Significativo em nível de 5\% de probabilidade de erro.

${ }^{\text {ns }}$ ão significativo.

diâmetro. O trado com $10 \mathrm{~cm}$ de diâmetro foi mais funcional para a coleta, o transporte e a separação das sementes de arroz-vermelho do solo, sendo necessárias 280, 55 e 31 ( $D=20 \%)$; 33, nove e seis ( $D=60 \%)$; e 13 , quatro e três $(\mathrm{D}=100 \%)$ amostras de solo, respectivamente para as infestações de 71, 282 e 498 sementes de arroz-vermelho por $\mathrm{m}^{2}$.

O número de amostras de solo necessário para estimar populações de sementes de arroz-vermelho é variável com o número médio de sementes no solo e com a precisão desejada. Para estudos com tratamentos em que há muita discrepância no nível de infestação do local, deve-se definir o número amostras necessárias pelo tratamento com menor infestação.

\section{REFERÊNCIAS}

AMBRÓSIO, L.A. et al. Assessment of the sample size to estimate the weed seedbank in soil. Weed Research, v.37, n.3, p.129137, 1997. Disponível em: <http://www3.interscience.wiley.com/ cgi-bin/fulltext/119946051/PDFSTART>. Acesso em: 12 ago. 2009. doi: 10.1046/j.1365-3180.1997.d01-22.x.

AMBRÓSIO, L.A. et al. Evaluation of sampling methods and assessment of the sample size to estimate the weed seedbank in soil, taking into account spatial variability. Weed Research, v.44, n.3, p.224-236, 2004. Disponível em: <http:// www3.interscience.wiley.com/cgi-bin/fulltext/118764661/ PDFSTART $>$. Acesso em 30 jun. 2009. doi: 10.1111/j.13653180.2004.00394.x.

CARMONA, R. Problemática e manejo de bancos de sementes de invasoras em solos agrícolas. Planta Daninha, v.10, n.1/2, p.5-16, 1992.

ROBERTS, H.A.; RICKETTS, M.E. Quantitative relationship between the weed flora after cultivation and the seed population in the soil. Weed Research, v.19, n.4, p.269-275, 1979. Disponível em: <http://www3.interscience.wiley.com/journal/ 119602458/abstract>. Acesso em 16 jul. 2009. doi: 10.1111/ j.1365-3180.1979.tb01537.x.

StEEL, R.G.D. et al. Principles and procedures of statistics: a biometrical approach. 3.ed. New York: McGraw Hill Book, 1997. 666p.

VIDOTTO, F. et al. A mathematical model to predict the population dynamics of Oryza sativa var. sylvatica. Weed Research, v.41, n.5, p.407-420, 2001. Disponível em:http:/ / w w w. se ma.r s.gov.br/s e m a / h t m l/ pd f/ Relatorio\%20Anual_completo_210207.pdf. Acesso em: 25 jun. 2009. doi: 10.1046/j.1365-3180.2001.00244.x. 